\title{
Development of a novel model to assess cognitive and memory impairment in rats after stroke
}

\section{R. Gal ${ }^{a}$, Y. Binyamin ${ }^{a}$, D. Natanel ${ }^{a}$, S. Gruenbaum ${ }^{b}$, B. Gruenbaum ${ }^{b}$, A. Zlotnik ${ }^{a}$.}

a Department of Anesthesiology, Soroka University Medical Center and the Faculty of Health Sciences, Ben-Gurion University of the Negev, Beer-Sheva, Israel.

b Departments of Anesthesiology, Yale University School of Medicine, New Haven, CT.

\section{Introduction}

Stroke is a leading cause of mortality and morbidity worldwide. Survivors of stroke frequently suffer from motor deficits, cognitive impairments, and neuropsychiatric complications that can result in disturbances in learning and special memory, impairing quality of life. Cerebrovascular disease and dementia are closely linked. Dementia has been described in about $30 \%$ of those with a history of stroke with a relative risk ranging from 3.6 to 5.8. The risk of dementia after stroke is high immediately after the insult and remains high even 25 years later. Interesting, elderly patients without a history of stroke but with cognitive decline have a higher risk of stroke. The pathophysiology of post-stroke dementia remains elusive and appears to be multifactorial rather than purely biological or psychosocial. Thus, valid animal models would contribute to the study its etiology and treatment. This study describes a novel rat model for post-stroke dementia using middle cerebral artery occlusion (MCAO).

\section{Methods}

40 Sprague Dawley rats (weight 350-450 g) were randomly assigned to receive $\mathrm{MCAO}$ using an uncoated 4-0 monofilament, or as a sham-control. An MRI was performed $24 \mathrm{hrs}$ post-surgery to evaluate the severity of

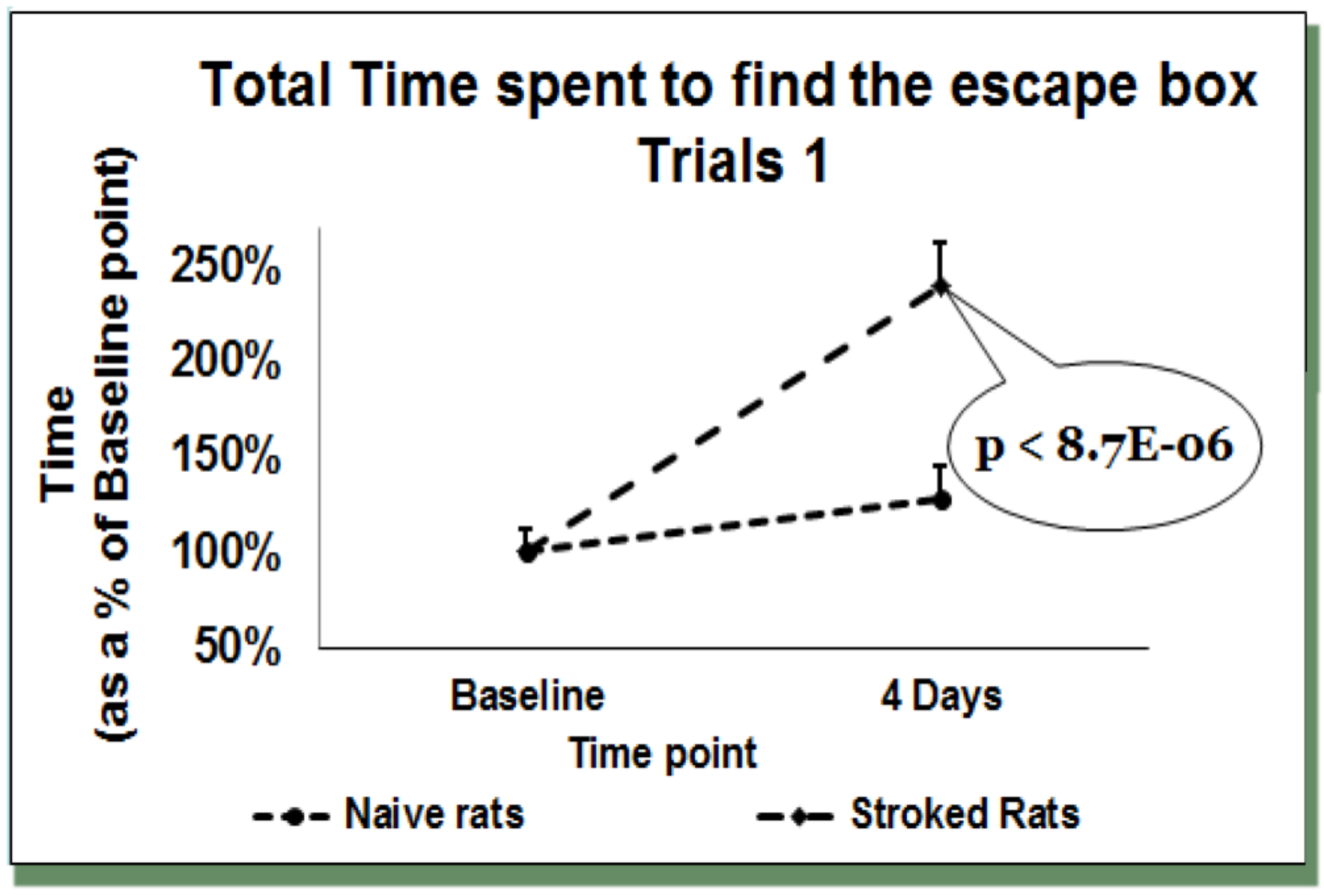

brain injury by measuring brain edema, infarct zone and blood brain barrier breakdown. On day 30 post-surgery the rats were introduced to the Barnes maze, an experimental tool to measure spatial learning and memory. After three days of training, time spent finding the escape box and the number of errors before finding the escape box was measured during two trails.

\section{Results}

On day 4 of the Barnes maze, there was a significant increase in the time spent finding the escape box and the number of errors before finding the escape box, during both trials in the post-stroke rats $(p<0.001)$. There was a significant increase in brain edema $(p<0.001)$, infarct zone $(p<0.001)$, and blood brain barrier breakdown $(p<0.001)$ in the post-stroke rats compared to controls. Rats from control group did not show any significant differences.

\section{Conclusions}

The findings of this study provide evidence that special learning and memory impairments following cerebral ischemia in rats are demonstrable. Animal models of post-stroke dementia may be useful in identifying the biological mechanisms underlying these symptoms and the development of therapeutic interventions.

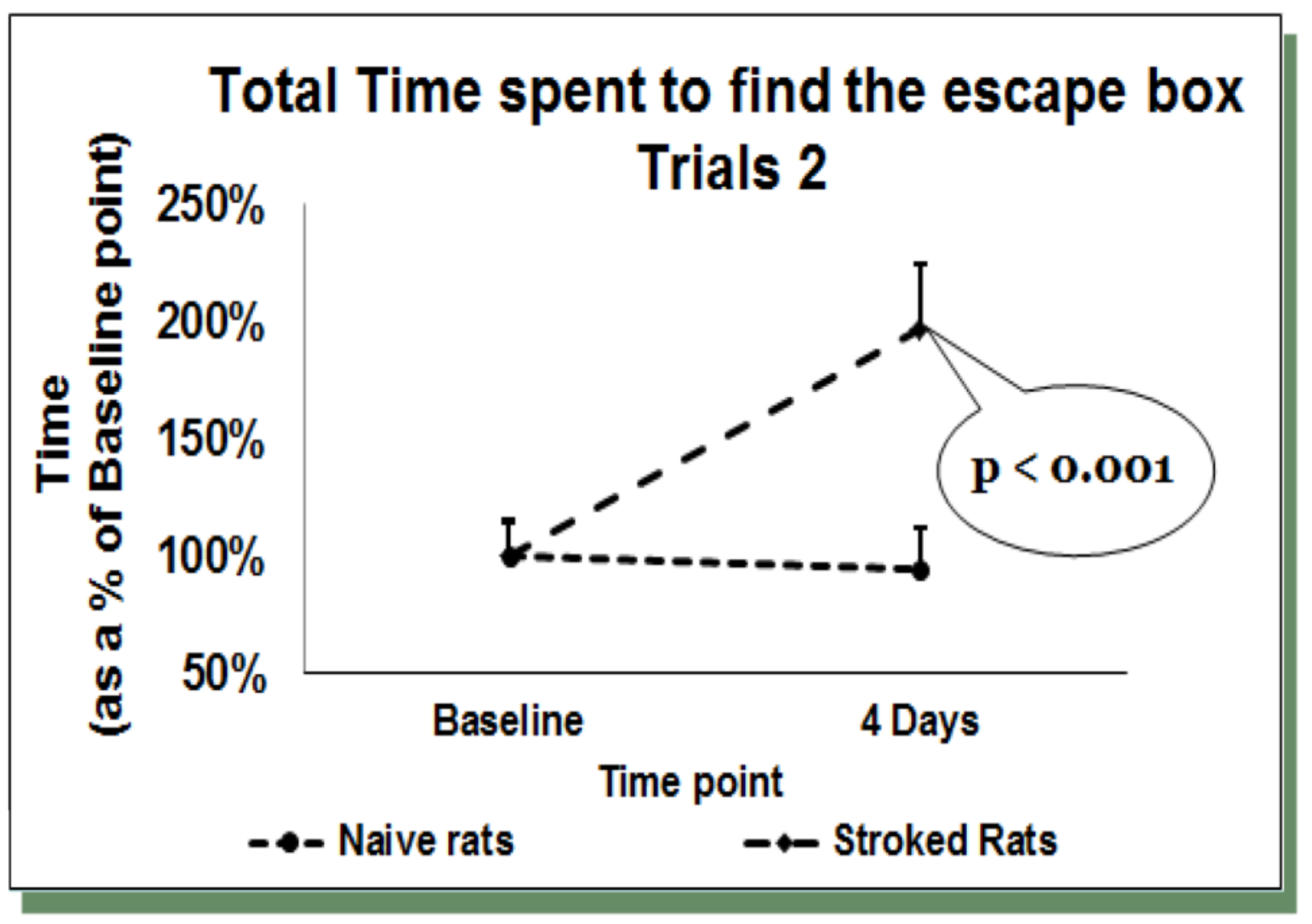

Copyright @ 2017 Gal ron | RonGal@clalit.org.il 